\title{
Representation of Abstract Quantitative Rules Applied to Spatial and Numerical Magnitudes in Primate Prefrontal Cortex
}

\author{
Anne-Kathrin Eiselt and Andreas Nieder \\ Animal Physiology, Institute of Neurobiology, University of Tuebingen, 72076 Tuebingen, Germany
}

Processing quantity information based on abstract principles is central to intelligent behavior. Neural correlates of quantitative rule selectivity have been identified previously in the prefrontal cortex (PFC). However, whether individual neurons represent rules applied to multiple magnitude types is unknown. We recorded from PFC neurons while monkeys switched between "greater than/less than" rules applied to spatial and numerical magnitudes. A majority of rule-selective neurons responded only to the quantitative rules applied to one specific magnitude type. However, another population of neurons generalized the magnitude principle and represented the quantitative rules related to both magnitudes. This indicates that the primate brain uses rule-selective neurons specialized in guiding decisions related to a specific magnitude type only, as well as generalizing neurons that respond abstractly to the overarching concept "magnitude rules."

\section{Introduction}

The ability to process quantity information to achieve internally maintained goals is central to intelligent behavior. One fundamental cognitive operation when dealing with magnitudes is to determine whether one magnitude is greater or less than another. This pertains to different types of magnitudes such as size and number. Rules applied to different magnitude types guide adaptive decisions in everyday situations of both humans and animals, be it foraging, mate choice, or social interactions (McComb et al., 1994; Wilson et al., 2012). Rule-based decisions on magnitude relations are hallmark operations in science and technology, but nonverbal precursors of this mental faculty are already found in nonhuman primates (Cantlon and Brannon, 2005; Bongard and Nieder, 2010).

The prefrontal cortex (PFC), operating at the apex of the cognitive hierarchy, is needed to implement abstract response strategies required for magnitude-related operations. Damage to the lateral PFC causes impairments of intricate mental processes related to numbers (Shallice and Evans, 1978; Smith and Milner, 1984) and other classes of abstract information (Milner, 1963; Badre et al., 2009). Consequently, PFC deficits resulting in executive dysfunctioning play a major role in neuropsychiatric disorders (Elliott, 2003; Fuster, 2008). Neurons in monkey PFC exhibit strategy-related activity (Genovesio et al., 2005; Mansouri et al., 2007; Tanji and Hoshi, 2008; Tsujimoto et al., 2012) and are

Received Dec. 20, 2012; revised March 8, 2013; accepted March 13, 2013.

Author contributions: A.-K.E. and A.N. designed research; A.-K.E. performed research; A.-K.E. analyzed data; A.-K.E. and A.N. wrote the paper.

This work was supported by the Bernstein Center for Computational Neuroscience, Tuebingen (Grant \#FKZ: 01GQ1002) and the Deutsche Forschungsgemeinschaft (Grant \#NI 618/4-1). We thank Torben Ott and Lena Veit for comments on this manuscript.

Correspondence should be addressed to Andreas Nieder, Department of Animal Physiology, Institute of Neurobiology, University of Tuebingen, 72076 Tuebingen, Germany. E-mail: andreas.nieder@uni-tuebingen.de.

DOI:10.1523/JNEUROSCI.5827-12.2013

Copyright $\odot 2013$ the authors $\quad 0270-6474 / 13 / 337526-09 \$ 15.00 / 0$ known to be involved in representing rules applied to specific images (White and Wise, 1999; Wallis et al., 2001; Buckley et al., 2009; Stoet and Snyder, 2009; Kamigaki et al., 2012) and numerosities (Bongard and Nieder, 2010). Neurophysiological studies so far have investigated rule coding only relative to a single specific category, such as same-different rules applied to pictures (Wallis et al., 2001) or "greater than/less than" rules applied to numerical quantity (Bongard and Nieder, 2010; Vallentin et al., 2012). In everyday situations, however, a limited number of ruleselective neurons have to cope with a variety of magnitude principles in a goal-directed way.

Here, we explored how neurons in monkeys performing a rule-switching task encode quantitative rules applied to visuospatial and visuonumerical magnitudes. One hypothesis suggests that the brain may treat the "greater than/less than" rules applied to both magnitudes as independent principles; in this case, rules related to numerical or spatial magnitudes would be encoded separately (Fig. 1A). Therefore, we would expect different populations of rule neurons for the two different magnitude types. Alternatively, the brain might emphasize the overarching concept "magnitude rules" in both protocols; after all, the length of a line and the number of items in a set both represent abstract magnitudes to which a general quantitative rule can be applied. Based on this hypothesis, neurons should represent the general instruction to choose the smaller amount of magnitude (shorter lines, fewer items) or the larger amount of magnitude (longer lines, more items), respectively, regardless of the precise type of magnitude (Fig. 1B). Of course, a mixture of these extreme hypotheses is also conceivable.

\section{Materials and Methods}

Animals. Data were collected from two male macaque monkeys (Macaca mulatta, monkey O: $8 \mathrm{~kg}$; monkey E: $4 \mathrm{~kg}$ ) that were cared for in accordance with the guidelines for animal experimentation approved by the Regierungspraesidium Tuebingen, Germany. Both male monkeys were 

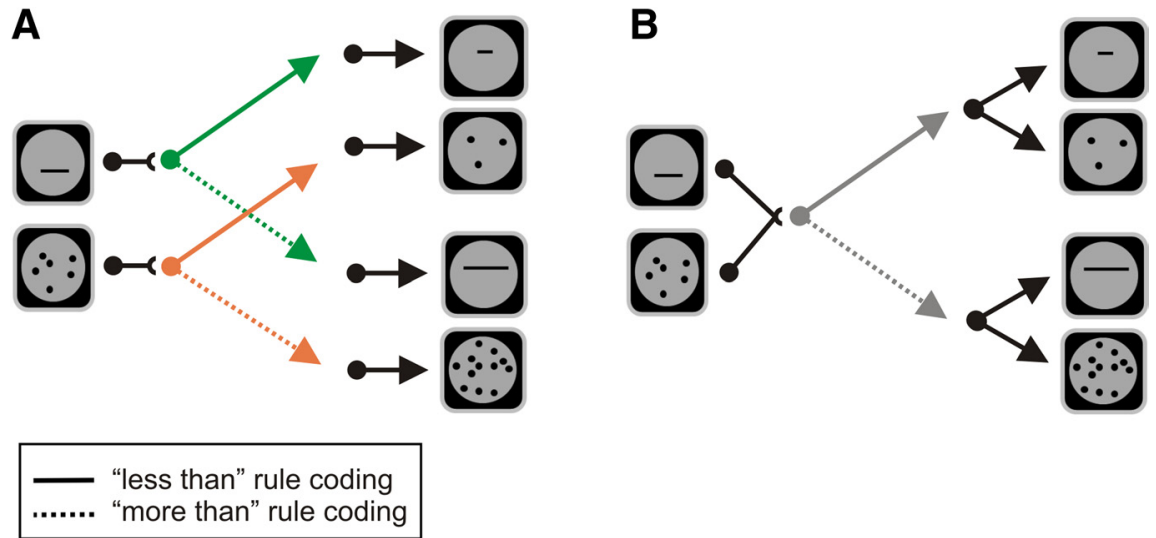

Figure 1. Rule-coding hypotheses. Two competing hypotheses can be formulated for the coding of quantitative rules applied to two magnitude types. $\boldsymbol{A}$, Hypothesis 1: the brain may treat different rules as independent principles and encode rules related to numerical or spatial magnitudes separately. $\boldsymbol{B}$, Hypothesis 2: the brain might emphasize the overarching principle "greater than/less than" in the two protocols and share neurons that represent the instruction to choose the smaller amount of magnitude (fewer items, shorter lines) or the larger amount of magnitude (more items, longer lines), respectively.

trained first on the numerosity comparison followed by the line-length comparisons. Both monkeys had experimental experience with numerical stimuli from previous experiments.

Behavioral protocol. The two monkeys learned to perform "greater than" and "less than" comparisons and flexibly switched between two different magnitude types (or categories). A monkey initiated a trial by grabbing a response bar and fixating a central fixation target (Fig. 2). After a fixation period of $500 \mathrm{~ms}$, a sample stimulus indicating the reference magnitude value was presented for $500 \mathrm{~ms}$. This sample period was followed by a $1000 \mathrm{~ms}$ delayl period during which the monkey had to remember the type and value of the sample display. Next, a rule cue (500 ms duration) instructed the monkey to apply either the "greater than" rule or the "less than" rule. The rule cue was then followed by a rule delay (delay2, 1000 ms duration) during which the monkey was informed about the rule at hand, but could not yet prepare a motor response. Then, a test 1 display appeared for $1200 \mathrm{~ms}$; here, the monkey was required to release the response bar if the "less than" rule had been cued and the displayed magnitude value was shorter/smaller than the reference value shown in the sample phase. Conversely, the monkey had to respond if the test1 display was longer/larger than the reference magnitude value if the "greater than" rule had been cued. This contingency was true in $50 \%$ of the randomized trials. In the other $50 \%$ of the trials, the magnitude value in the test 1 period did not match the cued rule; in this case, the monkey needed to maintain holding the response bar and only release it when the second test display (test2) was presented. Therefore, chance probability that either the display in the test 1 or test 2 period matched the rule instruction was 0.5. Trials were randomized and balanced across all relevant features (i.e., spatial and numerical magnitude, "greater than" and "less than" rule, rule cue modality, sample magnitude values). The monkey could only solve the task by assessing the magnitude value of the test displays relative to the two possible magnitude types and the three sample values together with the appropriate rule in any single trial. Eye movements were monitored with an infrared eye-tracking system (ISCAN) and monkeys were required to keep their gaze within $1.75^{\circ}$ of the fixation target until the test stimulus appeared.

Stimuli. Two different magnitude (or quantity) types were used: the length of lines and the number of items in a set (numerosities). These magnitudes were displayed in black on a gray background (diameter: $7^{\circ}$ of visual angle). For each magnitude, three different values were used. Line-length stimuli included line lengths of $1.2^{\circ}$ of visual angle (shorter test line length $=0.75^{\circ}$, longer test line length $\left.=2^{\circ}\right), 2^{\circ}\left(1.2^{\circ}, 3.2^{\circ}\right)$, and $3.2^{\circ}\left(2^{\circ}, 5^{\circ}\right)$, with line thicknesses ranging from $0.06^{\circ}$ to $0.36^{\circ}$. For the numerosity stimuli we presented sample numerosities 3 (smaller test numerosity $=1$, larger test numerosity $=6), 6(3,12)$, and $12(6,24)$. The dot size and position of each dot was randomized (diameter range: $0.3^{\circ}-$ $\left.1.3^{\circ}\right)$. We also tested both monkeys with a third magnitude type, the spatial frequency of sine-wave gratings. However, the sine-wave gratings applied to investigate the representation of the sensory magnitude "spa- tial frequency" were ambiguous; subjects could either judge the spatial frequency (the number of cycles per degree) or the wavelength of the stimuli, which is inversely related to spatial frequency. Thus, the "greater than/less than" rules were also ambiguous. We therefore excluded the spatial frequency magnitudes from the current study and focused on line length and numerosity stimuli, which are unequivocally defined.

All stimuli were generated anew using MATLAB (MathWorks) for each recording session to randomly vary the position and thickness/ size of the lines and numerosity dots. To further ensure that the monkeys solved the task based on the relevant quantitative information and to prevent the animals from exploiting low-level cues, other covarying features were controlled. Control magnitude stimuli were presented together with the standard stimuli described above in the same session. Dot density and total pixel area (i.e., contrast) across numerosities were controlled. Line-length stimuli were also equated for total pixel area. Both monkeys were tested with the same magnitude values. To dissociate the rule-related cellular responses from responses to sensory features of the rule cue, each rule was signified in two different sensory modalities: a red circle and a drop of water delivered with a white circle indicated the "greater than" rule, whereas a blue circle and no water delivered with a white circle cued the "less than" rule. Therefore, 96 specific trial conditions were tested in every session: 2 magnitude types (line length, numerosity), 3 magnitude sample values (e.g., 3, 6, and 12 dots for numerosity), 2 stimulus protocols (standard, control), 2 rules ("greater than", "less than"), 2 rule cues (color blue/red, with/without drops of water), and 2 trial types (test1 or test 2 correct).

To determine whether the monkeys generalized the different quantitative rules to magnitude values (samples) that had not been used during training, both monkeys performed the task with new sample stimuli for each magnitude type before electrophysiological recording. Generalization trials included the line length $1.3^{\circ}$ (shorter test line length $=0.8^{\circ}$, longer test line length $=2.08^{\circ}$ ) and the numerosity 4 (smaller test numerosity $=2$, larger test numerosity $=8$ ). Behavioral performance to generalization trials are shown in Figure 3.

Electrophysiological recordings. Extracellular single-cell activity was recorded from the right PFC (centered around the principal sulcus, Brodmann area 46 and Brodmann area 45) of two behaving rhesus monkeys using arrays of eight glass-coated tungsten microelectrodes (1 M $\Omega$ impedance) that were lowered into the brain each day. Custom made microdrives were used to lower the electrodes, with each drive controlling two electrodes through a plastic grid with $1 \mathrm{~mm}$ spacing. Neurons were recorded randomly; no prescreening for task-related activity was applied. This gave an unbiased sample of lateral PFC neurons. The recording chamber was stereotaxically placed and reconstructed using MRI images of both monkeys. Spikes were amplified and digitized using the Multichannel Acquisition Processor (Plexon) and then stored for offline sorting. All single units were sorted offline (Plexon).

Data analysis. Analysis of spike data and statistical tests were performed using MATLAB. Neuronal data were analyzed over a $700 \mathrm{~ms}$ window starting $500 \mathrm{~ms}$ after rule cue offset and pooled across stimulus (standard vs control) because we did not previously find any effect of these factors on behavioral and neuronal data (Tudusciuc and Nieder, 2007, 2009; e.g., Bongard and Nieder, 2010; Vallentin et al., 2012). A three-way ANOVA with the main factors of rule ("greater than" vs "less than"), rule cue (colors vs water/no water), and sample magnitude value (three magnitude values for each magnitude type) were performed separately for both magnitude types. We included neurons that had at least 2 trials for each condition for each of the two magnitude types, and mean firing rates $>1 \mathrm{~Hz}$. Rule-selective neurons showed a significant effect for 
main factor rule to one of the magnitude types and had no interaction with the other main factors (separately tested for each magnitude type, $p<0.01)$.

We investigated whether rule-selective neurons that encoded quantitative rules applied to more than one single magnitude type occurred more often than expected by chance. We used a binomial test to investigate the likelihood of the occurrence and preference congruence of generalizing cells. First, we assumed that both magnitude types are independent. We calculated the theoretical chance probability that a rule-selective neuron would encode rules related to both magnitude types by multiplying the proportions of the ruleselective neurons for each magnitude type. We then tested with a binomial test whether the observed probability of neurons encoding rules applied to more than one magnitude type was different from chance expectation.

To determine whether the rule generalizing cells ("generalists") preferred the same rule for both magnitude types (e.g., "greater than"), we evaluated rule preference congruency for each cell (i.e., how many cells show the same rule preference in both magnitude types). To investigate the coding quality of rule-selective neurons, we performed a receiver-operatingcharacteristic (ROC) analysis (Green and Swets, 1966) over the same $700 \mathrm{~ms}$ window used for the ANOVA for each magnitude type separately. To characterize the temporal evolution of individual neurons' rule selectivity, we computed a sliding window ROC analysis moved in $20 \mathrm{~ms}$ steps across $100 \mathrm{~ms}$ windows. The time points at which neurons significantly differentiated the "less than" from the "greater than" rule (or vice versa) were determined using a permutation test. For each time window, we calculated the null distribution by shuffling the distribution of firing rates for the "greater than/less than" conditions for each individual neuron (with 1000 repetitions, $p<0.05$ ) and assigned them anew to either category ("greater than" vs "less than"). If three consecutive time windows in a row showed significant $p$ values (i.e., exceeded the $95 \%$ upper threshold of the null distribution), we took the time point of the first significant analysis window as the neuron's latency for rule selectivity. Rule latency could not be determined for two cells with rule selectivity for line length and two cells with rule selectivity for numerosity. To compare latency differences between cells that generalized the rule to both magnitude types (generalists) and cells that encoded the rule only for a specific magnitude ("specialists"), we averaged across magnitude type to determine the mean area under the ROC curve (AUROC) values. For the generalists, we averaged the mean of the AUROC value of both magnitudes to obtain one AUROC value for each cell. To derive error AUROC values, we compared the activity of a "greater than" ("less than") neuron during correct "greater than" ("less than") trials with the activity of the same neuron when the monkey erroneously chose the smaller (larger) magnitude $(n=9)$.

\section{Results}

\section{Behavioral performance}

We trained monkeys to apply quantitative "greater than" and "less than" rules to two different classes of magnitudes: the length

B

\section{MAGNITUDE: LINE LENGTH}
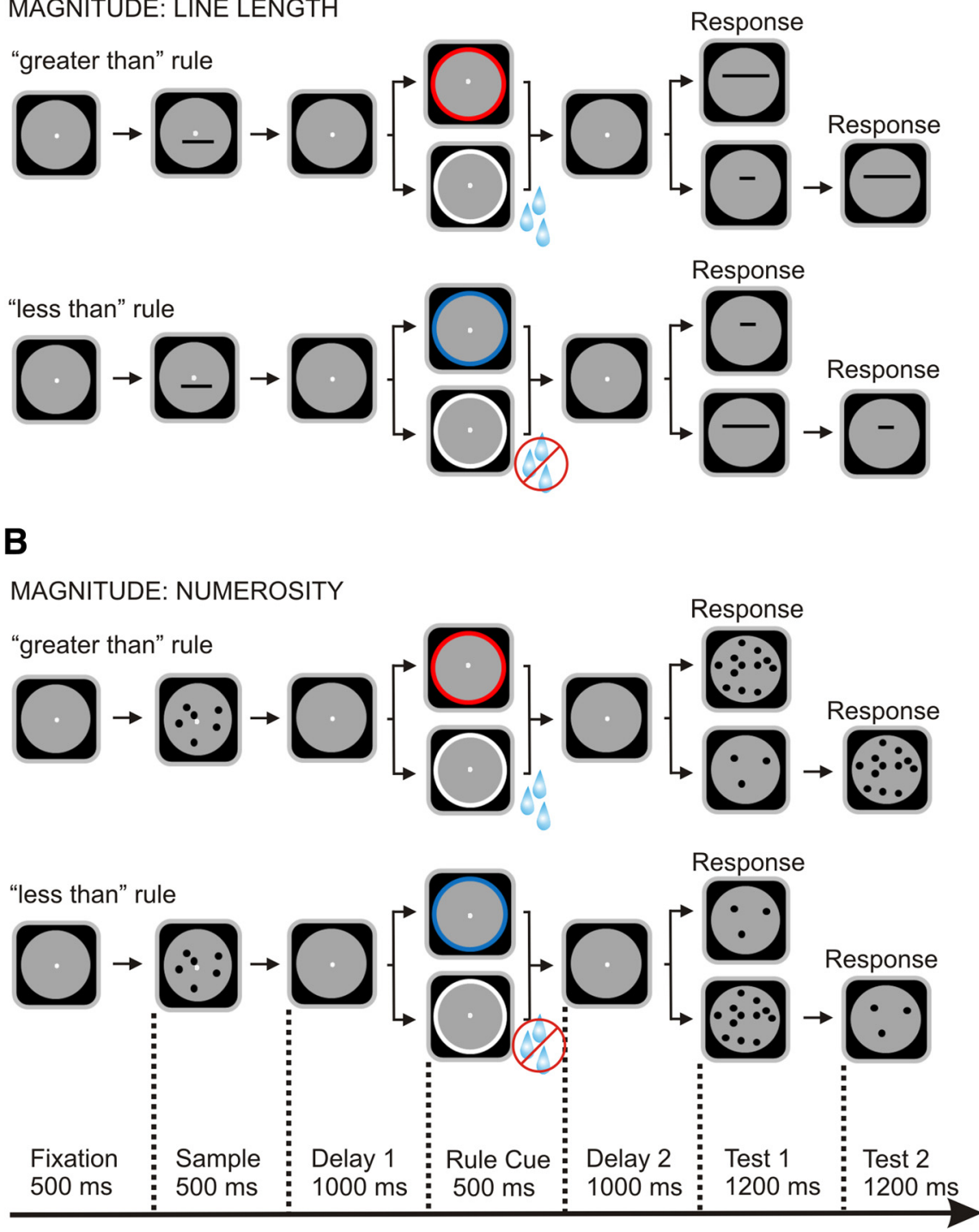

Figure 2. Task-switching protocol based on quantitative rules. $A$, Rules applied to magnitude line length. Monkeys grasped a lever and maintained central fixation throughout the trial until test phase. A sample line length was shown and followed by a working memory delay (delay1). Then, a rule cue indicated either the "greater than" or "less than" rule. Each rule was indicated by cues of two different sensory modalities (red circle or white circle with water for the "greater than" rule, blue circle or white circle without water for the "less than" rule). After a second delay (delay2), a test line length appeared and the monkeys were required to release the bar if the length of the test line was longer in "greater than" trials and to keep holding the bar if the test length was shorter. Conversely, the monkeys had to release the bar if the first test image displayed a shorter line length than the sample image in "less than" trials. $\boldsymbol{B}$, Rules applied to magnitude numerosity. The same behavioral protocol was used as in $\boldsymbol{A}$, but monkeys had to judge whether a test numerosity was greater or less than the sample numerosity based on the rule. In each session, magnitude types, magnitude values, rules, and rule cues were presented pseudorandomized.

of a line (continuous spatial magnitude) and numerosity (discrete numerical magnitude). The "greater than" rule required the monkeys to release the response bar if the first test display contained a magnitude longer/larger than that in the sample display, whereas the "less than" rule required the bar release if the magnitude of the first test display was shorter/smaller compared with the sample display. A rule cue (i.e., the "relational operator") displayed between sample and comparison magnitude informed the monkey about the valid rule to apply. The magnitude type, magnitude value, rule, and rule cue changed unpredictably for each individual trial. Figure $2 A, B$ shows the behavioral protocol for the two different magnitudes. 


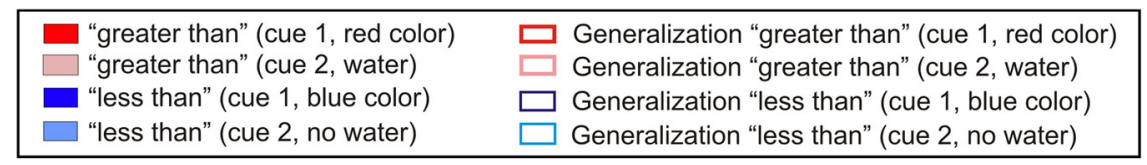

A

Monkey $\mathrm{E}$
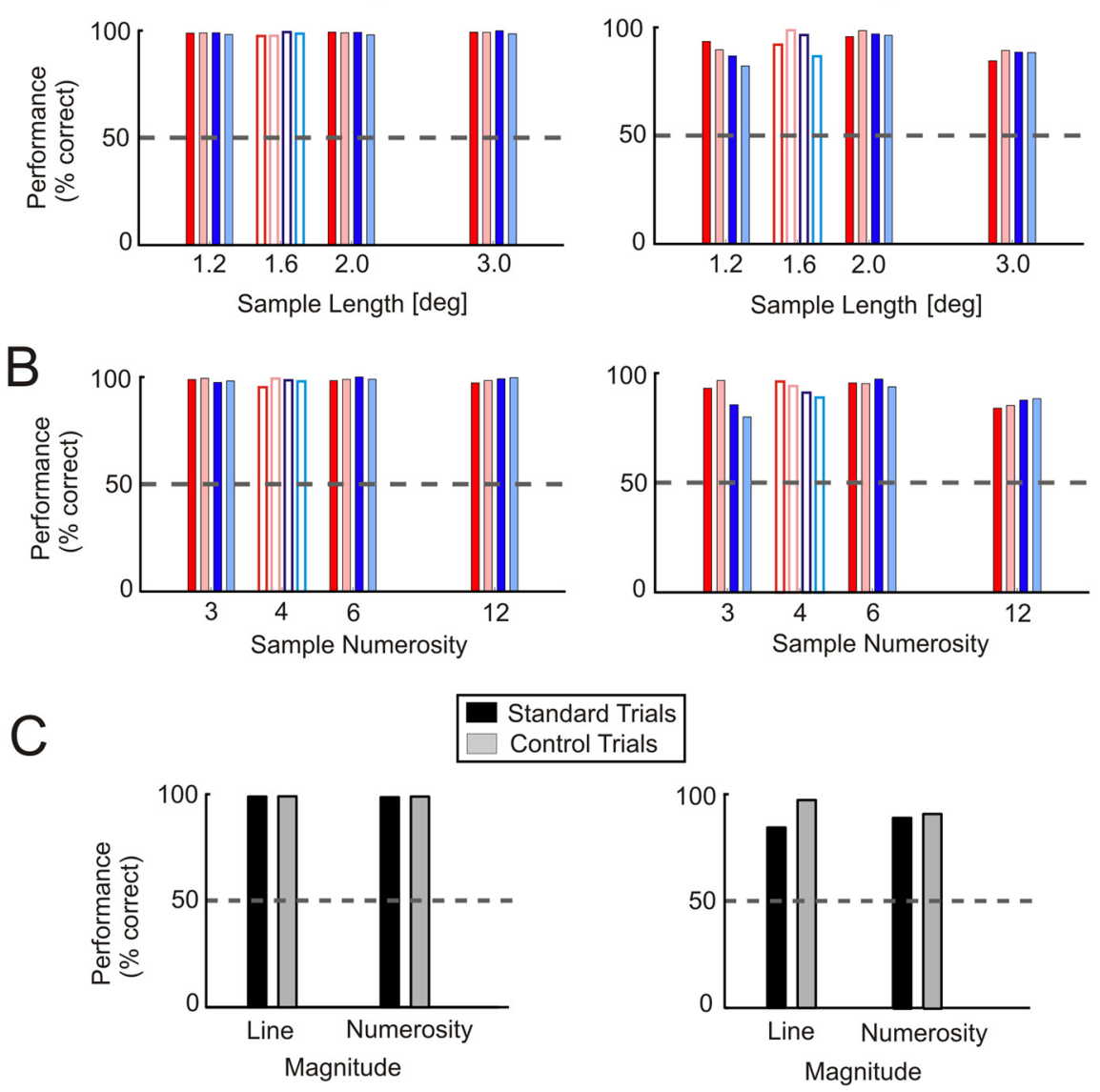

Standard Trials
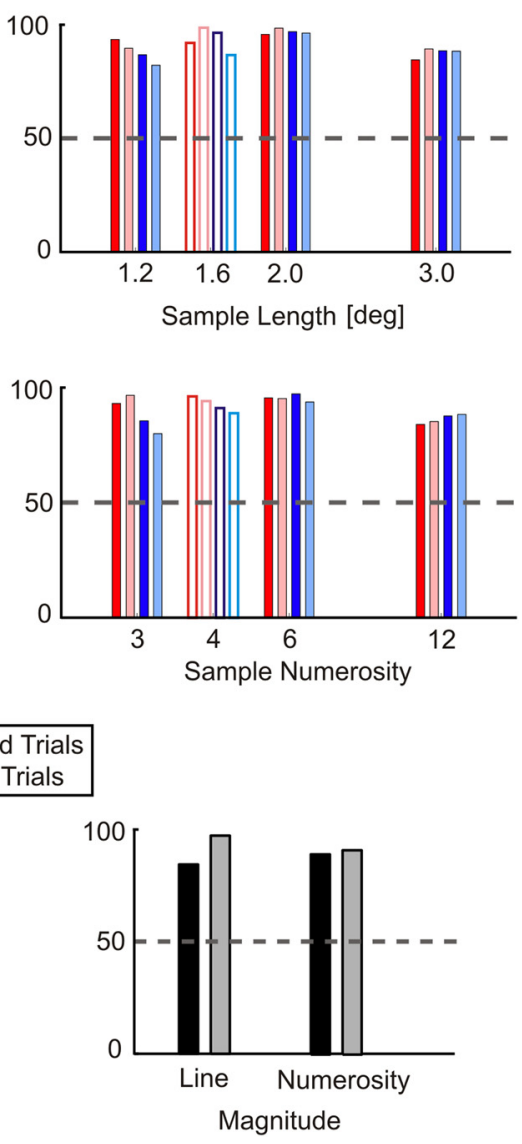

Figure 3. Behavioral performance. $A$, Performance of monkey $E$ (left) and monkey 0 (right) during electrophysiological recordings in the line-length protocol (standard and control protocols pooled). Columns represent percentage correct responses for the "greater than" and "less than" task. B, Performance for both monkeys in the numerosity protocol. C, Performance of both monkeys in control and standard trials for the quantitative rules applied to the two different magnitude types.

Both monkeys successfully learned the quantitative "greater than/less than" rules for the spatial and numerical magnitudes (Fig. 3). The monkeys were able to choose the smaller or larger magnitude value for each of the two magnitude types (each magnitude type was shown in three different magnitude values, e.g., short, medium, and long line length). The monkeys ignored the specific visual appearance of the two different magnitude displays and performed equally well in standard and control conditions shown for each magnitude type. Average correct performance in the standard condition for line length and numerosity stimuli were comparable to the control conditions (total dot area and dot density controls); all performance rates were significantly above chance level ( $p<0.001$, binomial test). Moreover, the animals' performance was comparable for the two rule cue modalities (red/blue vs water/no water) and for the two magnitude types (line length vs numerosity) (Fig. 3).

To ensure that the monkeys followed the quantitative principle regardless of the absolute value of the sample displays, we determined whether they generalized the "greater than/less than" rules to magnitude values within each magnitude domain that had not been presented in previous learning trials. Figure $3 A, B$ (open bars) shows that the performance in these generalization trials for the different magnitude types was virtually identical to that in the reinforced trained trials. This indicates that the monkeys followed an abstract "greater than/less than" principle regardless of the individual magnitude types and values.

\section{Neural activity to different magnitude rules}

We focused on the neural activity during the second delay (delay2; Fig. 2) because this is the period when the monkey is informed about the rule to apply, but cannot yet prepare a motor response due to the lacking comparison stimulus shown only in the subsequent test phase. The discharge rates were analyzed separately for the two different magnitude types during a $700 \mathrm{~ms}$ window starting $500 \mathrm{~ms}$ after cue offset by using a three-way ANOVA with main factors rule ("greater than" or "less than"), rule cue modality (visual or tactile), and magnitude value (smallest, median, or largest value per magnitude type) ( $p<$ 0.01 ). We recorded a total of 284 randomly selected neurons in the dorsolateral PFC around the principal sulcus. Overall, we found $24 \%$ (68/284) of all recorded cells with a significant rule effect (termed "rule-selective neurons" in the following) to line-length stimuli, numerosity stimuli, or both. [Neurons exhibiting only main effects of factor "sample" or factor "rule cue" in addition to a main effect of factor "rule" were included, but neurons with a significant interaction of other main factors with factor "rule" were excluded from the population of 68 ruleselective neurons (Wallis et al., 2001)]. A proportion of $19 \%$ of all recorded neurons $(54 / 284)$ were significantly selective to the rules applied to line length, whereas $13 \%$ of all recorded neurons (37/284) responded to the rules related to numerosity. The frequency of neurons responsive to rules applied to numerosity or line length did not differ ( $p>0.05, \chi^{2}$ test). A summary of the results obtained through the three-way ANOVA is listed in Table 1.

\section{Rule generalists versus rule specialists}

We next investigated whether rule-selective cells encoded quantitative rules to more than one magnitude type, and if so, whether the "greater than/less than" rule activity was congruent for both magnitudes. Of the 68 rule-selective cells, $66 \%$ (45/68) encoded only rules to one specific magnitude type (applied either to line length or numerosity), but were indifferent for the other magnitude. We called such rule-selective neurons rule specialists. Figure $4 D$ shows an example neuron only differentiating between the "greater than/less than" rule for line-length stimuli but not for numerosity stimuli and Figure $4 E$ depicts a neuron that was selective for the numerosity rule, but was indifferent for the line-length rule.

Interestingly, 34\% (23/68) of all rule-selective neurons encoded quantitative rules related to both magnitude types. Neu- 
Table 1. Percentages and numbers (in parenthesis) of all recorded neurons sorted by main factors and interactions

\begin{tabular}{lcccc}
\hline & $\begin{array}{l}\text { Number } \\
\text { specialists }\end{array}$ & $\begin{array}{l}\text { Line-length } \\
\text { specialists }\end{array}$ & Generalists & All \\
\hline Rule & $6 \%(17)$ & $10.9 \%(31)$ & $8.4 \%(24)$ & $25.4 \%(72)$ \\
Sample & $2.8 \%(8)$ & $5.3 \%(15)$ & $0.7 \%(2)$ & $8.8 \%(25)$ \\
Modality & $4.6 \%(13)$ & $3.5 \%(10)$ & $1.8 \%(5)$ & $9.9 \%(28)$ \\
Sample*Rule & $2.1 \%(6)$ & $1.1 \%(3)$ & $0 \%(0)$ & $3.2 \%(9)$ \\
Sample*Modality & $2.5 \%(7)$ & $1.1 \%(3)$ & $0 \%(0)$ & $3.5 \%(10)$ \\
Rule*Modality & $2.8 \%(8)$ & $2.1 \%(6)$ & $0.7 \%(2)$ & $5.6 \%(16)$ \\
Sample*Rule*Modality & $1.8 \%(5)$ & $1.8 \%(5)$ & $0 \%(0)$ & $3.5 \%(10)$ \\
Rule-selective cells w/0 & & & & \\
\multicolumn{1}{c}{ any rule interaction } & $4.9 \%(14)$ & $10.9 \%(31)$ & $8.1 \%(23)$ & $23.9 \%(68)$
\end{tabular}

Neurons are grouped based on their main factors and interactions in the three-way ANOVA $(p<0.01)$ during the analysis interval. Proportions are based on all recorded PFC neurons $(n=284)$. Note that for all reported analyses, we used rule-selective neurons that had no interaction with sample or rule cue modality.

rons encoding rules applied to both magnitude types can be expected by chance. For example, if rule 1 and rule 2 are represented by $15 \%$ of all neurons, chance predicts $2 \%$ of the neurons $(0.15 \times 0.15)$ to encode both rule 1 and rule 2 . However, we found more such rule generalists than would have been expected by chance ( $p<0.001$, binomial test). Even when considering only cells that exclusively had a rule effect and no other main effect, the proportion of rule generalists $(20 \%, 9 / 45)$ was still higher than expected by chance ( $p<0.001$, binomial test). Figure $4 B, C$ shows the activity of one of these abstract rule generalists encoding the "greater than/less than" rule to both magnitudes. The Venn diagram (Fig. 5) depicts the percentages and overlap of neuron populations encoding rules applied to line length, numerosity, or both. The generalizing neurons not only encoded the quantitative rules for both magnitudes, they also preferred the same rule; that is, a neuron discharging higher to the "greater than" rule in the numerosity protocol also preferred the "greater than" rule in the line-length task. The preferred rule was encoded congruently in almost all generalists $(22 / 23 ; p<0.001$, binomial test). Therefore, generalists that encode the "greater than" rule applied to line length also prefer the "greater than" rule applied to numerosity, and vice versa.

\section{Quality and temporal evolution of rule selectivity}

To quantify the quality of rule coding, we applied a ROC analysis to the neuronal activity in the same $700 \mathrm{~ms}$ time window at the end of the delay2 phase as used for the ANOVA. By convention, the discharge rates to the "less than" rule were the reference distribution. Therefore, values of AUROC could range from 0.5 (no rule information) to 0 (perfect discrimination between "greater than" and "less than" trials) for the "less than"-selective neurons, and 1.0 (perfect discrimination between "greater than" and "less than" trials) for the selective "greater than" neurons. A comparison of AUROC values of "greater than"- and "less than"-coding cells resulted in bimodal distributions for both magnitude types (Fig. 6A, $B$, top). Approximately half of the rule-selective neurons preferred the "greater than" rule (for line length: 34/54; for numerosity: 19/37), whereas the other half preferred the "less than" rule $(p>0.05$, binomial test).

To compare the quality of rule coding between the two magnitude types, the AUROC values of the "less than" neurons were subtracted from 1 ( 1 - AUROC of "less than" neurons) and pooled with the AUROC values of the "greater than" neurons for both magnitudes. The coding quality (based on the mean AUROC value) of the two rule-selective neuron populations ("greater than" and "less than" neurons) were similar for the line length ( 0.71 and 0.74 , respectively), and numerosity stimuli $(0.70$ and 0.71 , respectively) (for all comparisons $p>0.05$, MannWhitney $U$ test). Thus, both "greater than" and "less than" rules were represented equally frequent and with equal strength for the two magnitude types. The quality of rule coding between rule specialists versus rule generalists differed. Mean AUROC values were significantly higher for rule specialists (mean AUROC = $0.69)$ compared with rule generalists (mean AUROC $=0.67$ ) $(p<0.01$, Mann-Whitney $U$ test $)$.

In many neurons, rule selectivity evolved over time in the delay 2 phase. To investigate the temporal dynamics of rule selectivity for individual rule-selective neurons, we performed a sliding-window ROC analysis. To test statistically the time point at which neurons significantly encoded one rule over the other (i.e., rule latency) based on sliding AUROC values, we used a permutation analysis $(p<0.05$, permutation test; see data analysis for details). The neurons in Figure $6 A, B$ (bottom) are sorted according to rule latency. An increasing number of neurons became selective to the rules with increasing time after rule cue offset. There was no difference between rule latencies for rules applied to each magnitude type $(p>0.05$, Mann-Whitney $U$ test, latency could not be determined in four neurons), nor between rule generalists and rule specialists $(p>0.05$, MannWhitney $U$ test). In addition, latencies for each magnitude type within the generalists were not significantly different from each other $(p>0.05$, Wilcoxon signed-rank test).

\section{PFC activity predicts successful rule application}

Finally, we examined whether the activity of rule-selective neurons was relevant to task-switching behavior by comparing the neuronal discharge rates of individual rule-selective cells when monkeys completed a trial correctly with trials in which the monkey chose the wrong test display. If rule-selective neurons correlate with the monkeys' ability to choose the correct magnitude rule, then rule selectivity should be weakened whenever the monkeys make a mistake. Figure $7 A$ shows the neural activity of an example neuron's response in correct and error trials. The discharge to the preferred "greater than" rule was not only reduced during the delay2 phase, but was even reversed (the antipreferred "less than" rule elicited higher activity than the preferred "greater than" rule). We found that coding efficiency across the population of rule-selective neurons was significantly decreased in error trials (mean AUROC value $=0.58$ ) compared with correct trials (mean AUROC value $=0.70$ ) (Fig. $7 B ; p<0.01$, Wilcoxon signed-rank test). The AUROC values in error trials were not significantly different from 0.5 ( $p>0.05$, Wilcoxon signed-rank test). These findings indicate that rule-coding activity of PFC neurons related to magnitudes is behaviorally relevant.

\section{Discussion}

We examined how PFC neurons represent quantitative rules applied to two different types of magnitudes by recording lateral PFC neurons while monkeys flexibly switched between "greater than" and "less than" rules. These rules were related to either line length or numerosity. We found that the majority of ruleselective neurons encoded rules applied to an individual magnitude only. However, significantly more neurons than expected by chance also represented rules related to both magnitude types simultaneously.

\section{Rule-switching task applied to magnitudes}

To solve the rule-switching task, the monkeys had to rely on abstract principles of relations between magnitudes regardless of 


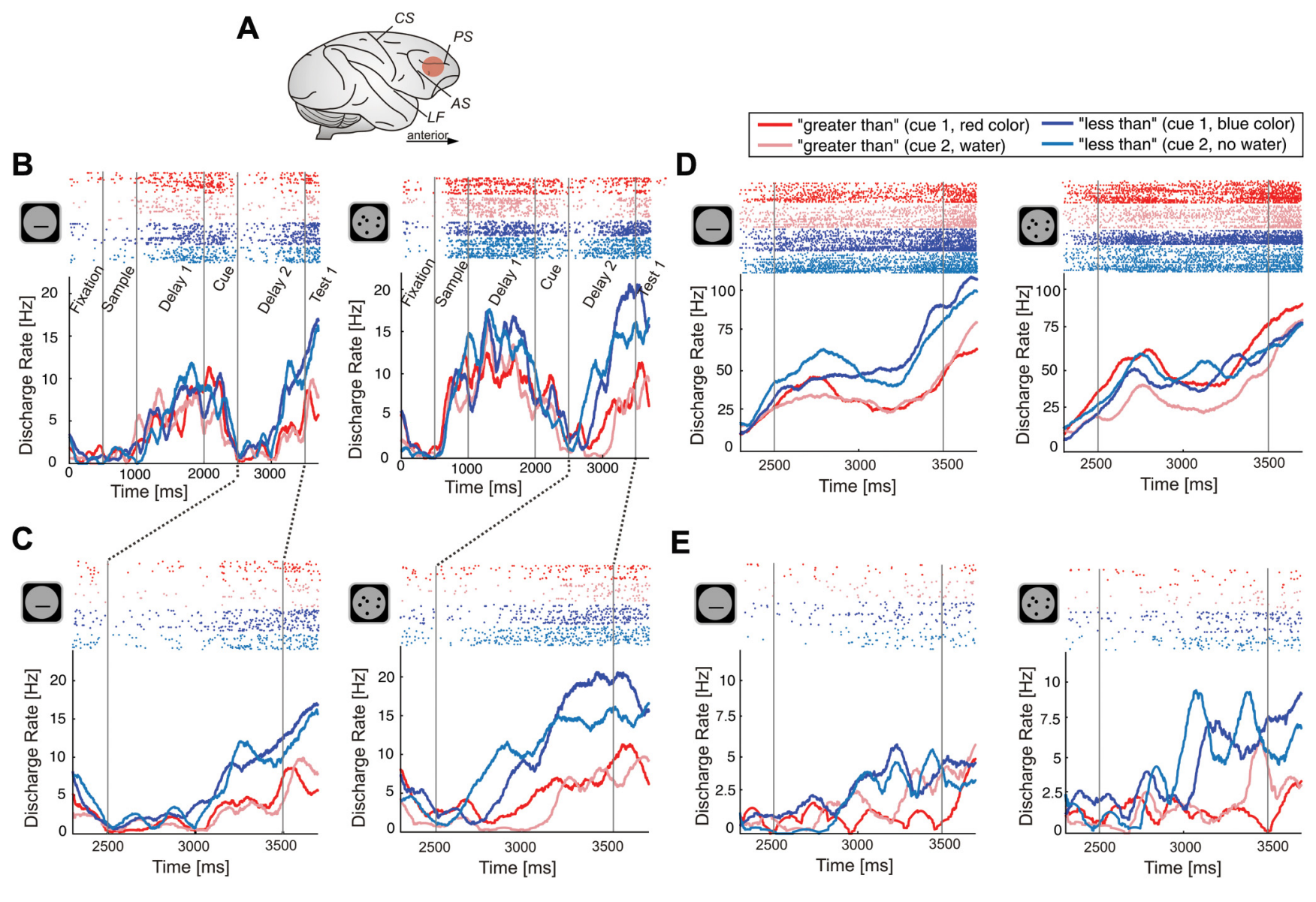

Figure 4. Responses of example neurons. $A$, Lateral view of a monkey brain showing the anatomical location of the recording site. We recorded from the right dorsolateral PFC of two monkeys. AS indicates arcuate sulcus; CS, central sulcus; LF, lateral fissure; PS, principal sulcus. B, C, Neuronal responses of a generalizing rule-selective example neuron preferring the "less than" rule in the line-length protocol (left) and the numerosity protocol (right). Top: Neuronal responses plotted as dot-raster histograms (each dot represents an action potential and spike trains are sorted and color coded according to the rules and rule cues). Bottom: Spike density functions (activity averaged over all trials and smoothed by a $150 \mathrm{~ms}$ Gaussian kernel). Rule selectivity was regardless of which cue signified the rule and which magnitude protocol was used. Only responses to correct trials are shown. $\boldsymbol{D}$, Neuron that differentiated between rules in trials with line-length stimuli as magnitude, but not in trials with numerosity stimuli. $\boldsymbol{E}$, Neuron that was rule selective when the rule was applied to numerosity, but not when the rule was applied to line length.

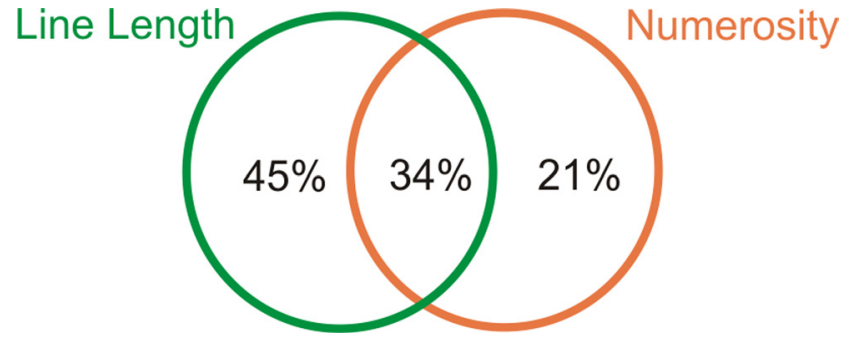

Figure 5. Proportions of rule-selective neurons. The Venn diagram depicts the percentages of rule-selective cells for either of the two magnitude types alone (rule specialists) or in conjunction (rule generalists) relative to all 68 rule-selective neurons.

the quantitative values of the sample and test displays and the rule cue modalities. We (Bongard and Nieder, 2010; Vallentin et al., 2012) and others (Cantlon and Brannon, 2005) have shown that monkeys grasp the relations between numerosities and follow rules applied to them in a goal-directed way. In the present study, however, the monkeys were required to apply "greater than/less than" rules to two different magnitude types simultaneously. The monkeys immediately generalized the "greater than/less than" rules to novel sample and test displays within each magnitude type (line length and numerosity). Therefore, behavior was based on an abstract magnitude principle.

\section{Magnitude codes in the brain}

Behavioral studies in humans and monkeys showed that the representations of different types of abstract magnitudes share many psychophysical features, often interfere with one another, and are thus not completely segregated (Henik and Tzelgov, 1982; Pinel et al., 2004; Merritt et al., 2010). Functional imaging studies in humans (Pinel et al., 2004; Castelli et al., 2006; Jacob and Nieder, 2009; Dormal et al., 2012) and monkeys (Onoe et al., 2001) suggest that anatomical vicinity or even a common magnitude system (Walsh, 2003) for the representation of abstract quantity in the parietal and prefrontal association cortices might be responsible for behavioral interference phenomena. Single-cell studies in monkeys confirmed the close anatomical vicinity of magnitude representations, both in the posterior parietal cortex (Sawamura et al., 2002; Nieder and Miller, 2004; Nieder et al., 2006; Tudusciuc and Nieder, 2007) and the dorsolateral PFC (Nieder et al., 2002; Tudusciuc and Nieder, 2009; Genovesio et al., 2011). Different magnitude types are encoded by functionally overlapping, anatomically intermingled groups of quantity detectors (Tudusciuc and Nieder, 2007, 2009; Genovesio et al., 2012), resulting in distributed but overlapping neural coding of magnitude dimensions. In the realm of numerical competence, abstract numerosity is encoded by neurons that integrate numerical information across spatiotemporal presentation formats (Nieder et al., 2006) and visuoauditory modalities (Nieder, 2012). 


\section{PFC neurons encoding} quantitative rules

Important as it is as a first step, the mere representation of magnitude does not on its own constitute a cognitive advantage. After quantities are extracted from sensory input, specifically in the putative parietal semantic quantification system in the fundus of the intraparietal sulcus (Nieder and Dehaene, 2009; Nieder, 2013), they need to be further processed. As a hallmark of executive functioning (Miller and Cohen, 2001; Fuster, 2008; Stoet and Snyder, 2009), different sources of external and internal information need to be integrated before quantities can successfully influence behavior. To that aim, information needs to be conveyed to the PFC operating on a higher hierarchy level. Flexible grouping of information into behaviorally meaningful categories is a cardinal function of PFC (Rainer et al., 1998; Cromer et al., 2010; Roy et al., 2010). In agreement with these findings, quantity categories are represented more abstractly in the dorsolateral PFC (Nieder and Miller, 2004; Diester and Nieder, 2007; Nieder, 2009; Vallentin et al., 2012, Nieder, 2012).

Here we report that $34 \%$ of ruleselective PFC neurons (23/68) encode quantitative rules applied to two different magnitude types simultaneously. The analyzed rule activity in the delay 2 phase represents the neuronal signal of the abstract quantitative principle (or rule-based decision) applied to the two magnitude types because we excluded neurons that had statistical interactions with either the rule cue or the sample value. In addition, this activity cannot be a preparatory motor signal either because the monkey lacks the necessary information of the comparison stimulus only displayed in the test 1 period to prepare a response. Tsujimoto et al. (2012) found cells in PFC that encode strategies (stay and shift) in a modalityspecific manner. However, we (Bongard and Nieder, 2010; Vallentin et al., 2012) and others (Wallis et al., 2001) have shown that the rule is encoded abstractly in rule-switching tasks (i.e., in a manner independent of rule cue modality). Error trial analyses emphasized the behavioral significance of rule-related activity for correct "greater than/less than" choices. The coding quality (reflected by AUROC values) in the delay2 period was significantly reduced whenever the monkeys made response errors. Therefore, the monkeys' behavior was error-prone whenever the neurons' activity did not properly differentiate between rules. The causal role of the dorsolateral PFC in task shifting based on rules has also been demonstrated in lesion (Buckley et al., 2009)

A represent SEM. ${ }^{*} p<0.01$.
Line Length Rule

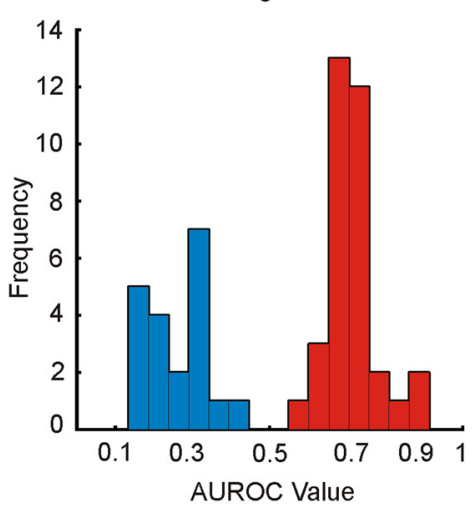

B

Numerosity Rule
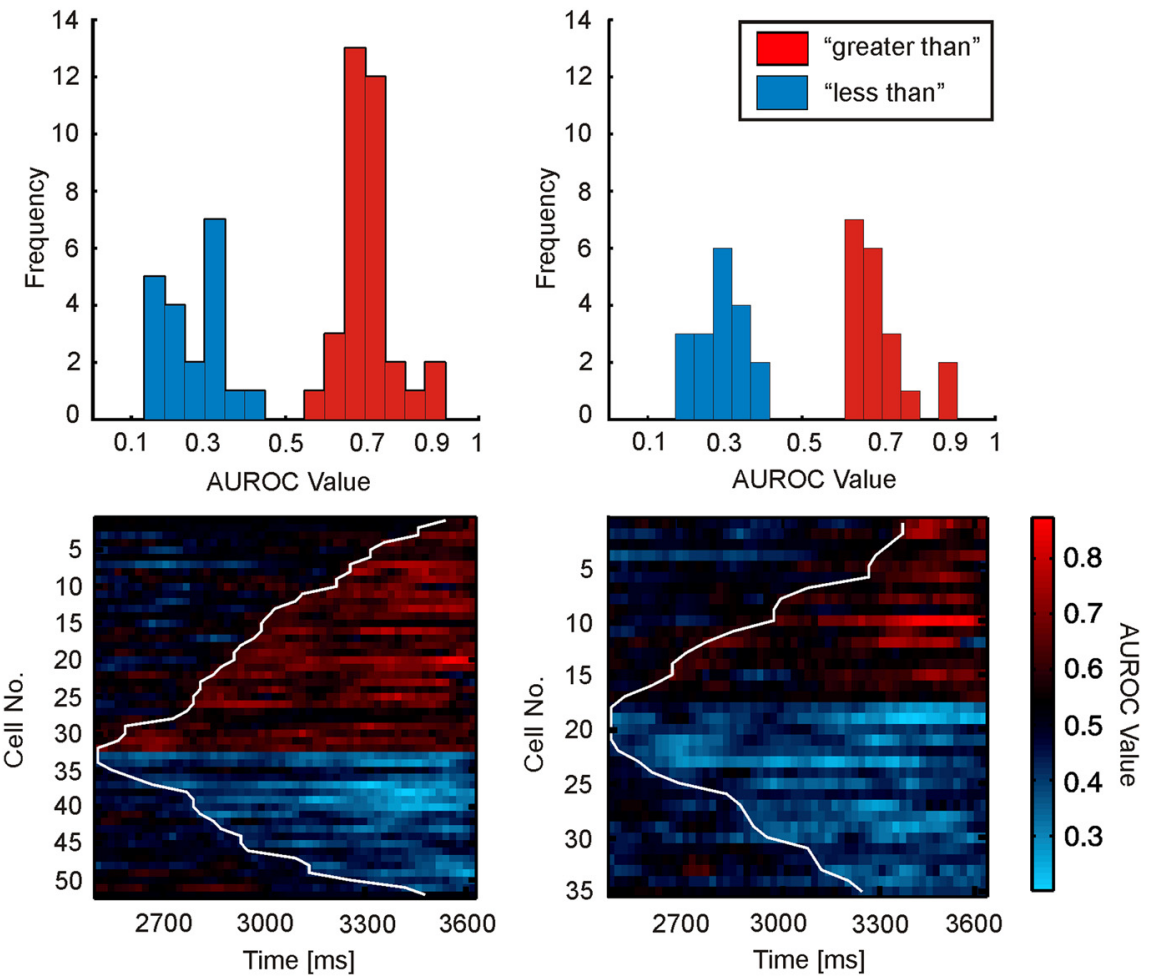

Figure 6. Quality and temporal evolution of rule selectivity. $A$, Top: Frequency histogram of AUROC values of neurons encoding abstract quantitative rules for line-length stimuli during correct trials. Bottom: Temporal evolution of rule-selective signals in the delay2 period for the line-length magnitude rule-selective neurons. Each row in the color map represents the time course of rule-selective coding (AUROC value) for an individual neuron. Neurons are sorted according to their rule preference ("greater than" rule in red with AUROC values larger than 0.5 and "less than" rule in blue with AUROC values smaller than 0.5 ) and latency of significant rule selectivity (white line in the color map). $\boldsymbol{B}$, Frequency histogram (top) and temporal evolution of AUROC values (bottom) of rule-selective neurons selective to the numerosity rules.

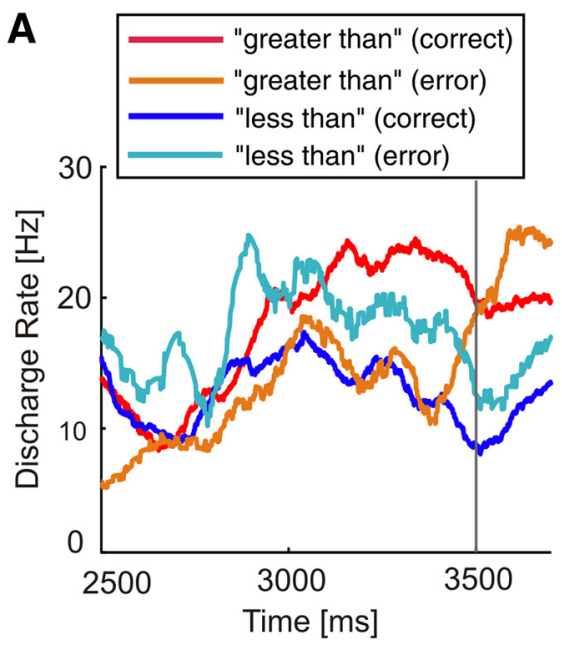

B

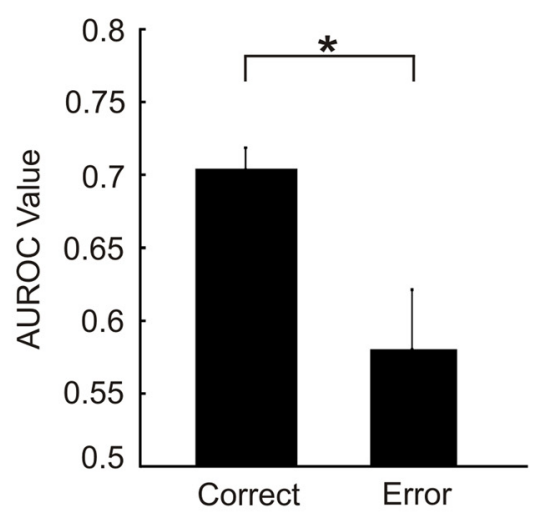

Figure 7. Behavioral relevance of rule-selective activity. $A$, Discharge rates of one example neuron during the delay2 period for correct and error trials. $\boldsymbol{B}$, Mean AUROC values for error trials compared with correct trials (pooled across magnitudes). Error bars

and reversible inactivation studies (Kamigaki et al., 2012); inactivation of dorsolateral PFC selectively impairs performance on behavioral shifting.

In the present study, $13 \%$ of all recorded neurons selectively encoded the quantitative rule applied to numerosity. 
This fraction is in good agreement with our earlier findings of $19 \%$ of rule-selective neurons in the PFC (Bongard and Nieder, 2010; Vallentin et al., 2012). In addition, a similar fraction (19\% of all neurons) represented the "greater than/less than" rule related to line length. The majority of rule-selective neurons $(66 \%)$ were rule specialists that only encoded the quantitative "greater than/less than" rules applied to a single, specific (spatial or numerical) magnitude type. Probability assumptions predict that single rule-selective neurons can be expected to encode rules to both magnitude types simply by chance. However, we found that the probability of neurons encoding rules applied to both tested magnitudes (34\%) was more than expected by chance.

These rule generalists showed significantly lower AUROC values for the "greater than" and "less than" rule, which might indicate that rule specialists have better rule-coding qualities than rule generalists. Cromer et al. (2010) found stronger coding quality in PFC neurons representing two independent category distinctions rather than only a single one during a delayed match-to-category task, which is in contrast to our results. We found no difference in coding latency between rule generalists and rule specialists.

Our data suggest a mixed representation of the outlined extreme hypotheses. The brain reserves a majority of rule specialists to represent the "greater than/less than" rules applied to a single magnitude type as independent principles. This is helpful in processing the specific magnitude category at hand in a goal-directed way. Conversely, the population of rule generalists indicates that also the overarching concept "magnitude rule" is emphasized. Because these quantitative rule representations are abstracted beyond the specific details of quantities, they can easily be generalized and adapted to new circumstances.

\section{Outlook}

The PFC does not process rule-related information in isolation; rather, strategic behavior seems to require a larger network of cortical and even subcortical areas (Muhammad et al., 2006). The premotor cortex, for example, seems to reflect abstract rules even more strongly than the PFC (Wallis and Miller, 2003; Vallentin et al., 2012). In addition, neurons in other parts of the frontal lobe, such as the anterior cingulate cortex (Johnston et al., 2007) or cingulate motor areas (Vallentin et al., 2012), are also engaged during rule-guided tasks. A better understanding of the respective contributions of different brain structures in encoding quantitative rules will require direct comparisons of activity patterns in candidate regions.

\section{References}

Badre D, Hoffman J, Cooney JW, D’Esposito M (2009) Hierarchical cognitive control deficits following damage to the human frontal lobe. Nat Neurosci 12:515-522. CrossRef Medline

Bongard S, Nieder A (2010) Basic mathematical rules are encoded by primate prefrontal cortex neurons. Proc Natl Acad Sci U S A 107:2277-2282. CrossRef Medline

Buckley MJ, Mansouri FA, Hoda H, Mahboubi M, Browning PG, Kwok SC, Phillips A, Tanaka K (2009) Dissociable components of ruleguided behavior depend on distinct medial and prefrontal regions. Science 325:52-58. CrossRef Medline

Cantlon JF, Brannon EM (2005) Semantic congruity affects numerical judgments similarly in monkeys and humans. Proc Natl Acad Sci U S A 102: 16507-16511. CrossRef Medline

Castelli F, Glaser DE, Butterworth B (2006) Discrete and analogue quantity processing in the parietal lobe: a functional MRI study. Proc Natl Acad Sci U S A 103:4693-4698. CrossRef Medline
Cromer JA, Roy JE, Miller EK (2010) Representation of multiple, independent categories in the primate prefrontal cortex. Neuron 66:796807. CrossRef Medline

Diester I, Nieder A (2007) Semantic associations between signs and numerical categories in the prefrontal cortex. PLoS Biol 5:2684-2695. CrossRef Medline

Dormal V, Dormal G, Joassin F, Pesenti M (2012) A common right frontoparietal network for numerosity and duration processing: an fMRI study. Hum Brain Mapp 33:1490-1501. CrossRef Medline

Elliott R (2003) Executive functions and their disorders. Br Med Bull 65:49_ 59. CrossRef Medline

Fuster J (2008) The prefrontal cortex, Ed 4. London: Academic.

Genovesio A, Brasted PJ, Mitz AR, Wise SP (2005) Prefrontal cortex activity related to abstract response strategies. Neuron 47:307-320. CrossRef Medline

Genovesio A, Tsujimoto S, Wise SP (2011) Prefrontal cortex activity during the discrimination of relative distance. J Neurosci 31:3968-3980. CrossRef Medline

Genovesio A, Tsujimoto S, Wise SP (2012) Encoding goals but not abstract magnitude in the primate prefrontal cortex. Neuron 74:656-662. CrossRef Medline

Green DM, Swets JA (1966) Signal detection theory and psychophysics. New York: Wiley.

Henik A, Tzelgov J (1982) Is three greater than five: the relation between physical and semantic size in comparison tasks. Mem Cognit 10:389395. CrossRef Medline

Jacob SN, Nieder A (2009) Tuning to non-symbolic proportions in the human frontoparietal cortex. Eur J Neurosci 30:1432-1442. CrossRef Medline

Johnston K, Levin HM, Koval MJ, Everling S (2007) Top-down controlsignal dynamics in anterior cingulate and prefrontal cortex neurons following task switching. Neuron 53:453-462. CrossRef Medline

Kamigaki T, Fukushima T, Tamura K, Miyashita Y (2012) Neurodynamics of cognitive set shifting in monkey frontal cortex and its causal impact on behavioral flexibility. J Cogn Neurosci 24:2171-2185. CrossRef Medline

Mansouri FA, Buckley MJ, Tanaka K (2007) Mnemonic function of the dorsolateral prefrontal cortex in conflict-induced behavioral adjustment. Science 318:987-990. CrossRef Medline

McComb K, Packer C, Pusey A (1994) Roaring and numerical assessment in contests between groups of female lions, Panthera leo. Animal Behavior 47:379-387. CrossRef

Merritt DJ, Casasanto D, Brannon EM (2010) Do monkeys think in metaphors? Representations of space and time in monkeys and humans. Cognition 117:191-202. CrossRef Medline

Miller EK, Cohen JD (2001) An integrative theory of prefrontal cortex function. Annu Rev Neurosci 24:167-202. CrossRef Medline

Milner B (1963) Effects of different brain lesions on card sorting. Arch Neurol 9:100-110.

Muhammad R, Wallis JD, Miller EK (2006) A comparison of abstract rules in the prefrontal cortex, premotor cortex, inferior temporal cortex, and striatum. J Cogn Neurosci 18:974-989. CrossRef Medline

Nieder A (2009) Prefrontal cortex and the evolution of symbolic reference. Curr Opin Neurobiol 19:99-108. CrossRef Medline

Nieder A (2012) Supramodal numerosity selectivity of neurons in primate prefrontal and posterior parietal cortices. Proc Natl Acad Sci U S A 109: 11860-11865. CrossRef Medline

Nieder A (2013) Coding of abstract quantity by 'number neurons' of the primate brain. J Comp Physiol A Neuroethol Sens Neural Behav Physiol 199:1-16. CrossRef Medline

Nieder A, Dehaene S (2009) Representation of number in the brain. Annu Rev Neurosci 32:185-208. CrossRef Medline

Nieder A, Miller EK (2004) A parieto-frontal network for visual numerical information in the monkey. Proc Natl Acad Sci U S A 101:74577462. CrossRef Medline

Nieder A, Freedman DJ, Miller EK (2002) Representation of the quantity of visual items in the primate prefrontal cortex. Science 297:17081711. CrossRef Medline

Nieder A, Diester I, Tudusciuc O (2006) Temporal and spatial enumeration processes in the primate parietal cortex. Science 313:1431-1435. CrossRef Medline

Onoe H, Komori M, Onoe K, Takechi H, Tsukada H, Watanabe Y (2001) 
Cortical networks recruited for time perception: a monkey positron emission tomography (PET) study. Neuroimage 13:37-45. CrossRef Medline

Pinel P, Piazza M, Le Bihan D, Dehaene S (2004) Distributed and overlapping cerebral representations of number, size, and luminance during comparative judgments. Neuron 41:983-993. CrossRef Medline

Rainer G, Asaad WF, Miller EK (1998) Selective representation of relevant information by neurons in the primate prefrontal cortex. Nature 393: 577-579. CrossRef Medline

Roy JE, Riesenhuber M, Poggio T, Miller EK (2010) Prefrontal cortex activity during flexible categorization. J Neurosci 30:8519-8528. CrossRef Medline

Sawamura H, Shima K, Tanji J (2002) Numerical representation for action in the parietal cortex of the monkey. Nature 415:918-922. CrossRef Medline

Shallice T, Evans ME (1978) The involvement of the frontal lobes in cognitive estimation. Cortex 14:294-303. Medline

Smith ML, Milner B (1984) Differential effects of frontal-lobe lesions on cognitive estimation and spatial memory. Neuropsychologia 22:697-705. CrossRef Medline

Stoet G, Snyder LH (2009) Neural correlates of executive control functions in the monkey. Trends Cogn Sci 13:228-234. CrossRef Medline

Tanji J, Hoshi E (2008) Role of lateral prefrontal cortex in executive behavioral control. Physiol Rev 88:37-57. CrossRef Medline

Tsujimoto S, Genovesio A, Wise SP (2012) Neuronal activity during a cued strategy task: comparison of dorsolateral, orbital, and polar prefrontal cortex. J Neurosci 32:11017-11031. CrossRef Medline

Tudusciuc O, Nieder A (2007) Neuronal population coding of continuous and discrete quantity in the primate posterior parietal cortex. Proc Natl Acad Sci U S A 104:14513-14518. CrossRef Medline

Tudusciuc O, Nieder A (2009) Contributions of primate prefrontal and posterior parietal cortices to length and numerosity representation. J Neurophysiol 101:2984-2994. CrossRef Medline

Vallentin D, Bongard S, Nieder A (2012) Numerical rule coding in the prefrontal, premotor and posterior parietal cortices of macaques. J Neurosci 32:6621-6630. CrossRef Medline

Wallis JD, Miller EK (2003) From rule to response: neuronal processes in the premotor and prefrontal cortex. J Neurophysiol 90:17901806. CrossRef Medline

Wallis JD, Anderson KC, Miller EK (2001) Single neurons in prefrontal cortex encode abstract rules. Nature 411:953-956. CrossRef Medline

Walsh V (2003) A theory of magnitude: common cortical metrics of time, space and quantity. Trends Cogn Sci 7:483-488. CrossRef Medline

White IM, Wise SP (1999) Rule-dependent neuronal activity in the prefrontal cortex. Exp Brain Res 126:315-335. CrossRef Medline

Wilson ML, Kahlenberg SM, Wells M, Wrangham RW (2012) Ecological and social factors affect the occurrence and outcomes of intergroup encounters in chimpanzees. Animal Behavior 83:277-291. CrossRef 